\title{
Prediction of Vapour-Liquid Equilibrium for n-alkane Fluids
}

\section{BINAY PRAKASH AKHOURI ${ }^{*}$, KHURSHID AKHTAR ${ }^{2}$ and TEJ PRAKASH AKHOURI}

\author{
'Department of Physics, Birsa college, Khunti-835210, Jharkhand, India. \\ ${ }^{2}$ Post Graduate Department of Chemistry, Ranchi College, Ranchi-834008, Jharkhand, India. \\ *Corresponding author E-Mail address: binayakhouri@yahoo.in
}

http://dx.doi.org/10.13005/ojc/320161

(Received: December 21, 2015; Accepted: March 14, 2016)

\begin{abstract}
Analysis of the equations of state of the hard convex body chain and hard spheres has been done for predicting the vapor liquid equilibrium of simple fluids of $n$-alkanes. The repulsive part of the Boublik equation of state for the hard convex body chain has been found as an equivalent alternative either for the well known Carnahan-Starling repulsive term or the established van der Waals repulsive part of hard spheres equations of state. The attractive parts of these equations of state have the similar form as that of the van der Waals and are obeying the power-law temperature dependency. Add-on separation method of compressibility factor has been used for these equations of state. The simulated data for VLE densities from these equations of state are found to agree well with the available experimental data for $n$-alkane fluids.
\end{abstract}

Key words: Equations of state; Vapour liquid equilibrium; Critical parameters; Hard convex body chain.

\section{INTRODUCTION}

An equation of state (EoS) is an analytical expression relating pressure to the volume and temperature. The expression of an equation of state is used ${ }^{1-3}$ to describe the volumetric behavior, the vapor-liquid equilibria (VLE), and the thermal properties of pure substances and mixtures. Numerous EoS have been proposed to represent the phase behavior of pure substances and mixtures in the gas and liquid states since van der Waals introduced his expression in 1873. The van der Waals equation provides a qualitatively correct description of the critical point and vapor-liquid equilibrium envelope but for practical applications it is too crude. Nonetheless, it formed a certain basis for developing dozens of empirical equations of state (EoS) used in engineering applications ${ }^{4-6}$.

The attraction parameter ' $a$ ' of van der Waals needed to be made a function of temperature before any cubic EoS was able to do a better job of quantitatively matching experimental data. This was a realization that van der Waals himself had suggested, but no actual functional dependency had been introduced until the Redlich-Kwong ${ }^{5}$ 
EoS. The Redlich-Kong equation of state improved the accuracy of the van der Waals by proposing a temperature dependence attractive term. Thus, the compressibility factors of most empirical equations of state can be expressed as the sum of a temperature-independent term and a temperaturedependent term. Using this functional dependency of temperature in attractive term, the improvement in results for the vapor-liquid equilibrium properties is found significant when the simple but very precise Carnahan-Starling equation has been used. Since these equations of state are cubic in volume, so that the van der Waals concept is retained.

Molecular simulation reveals that the inaccuracies of attractive and repulsive terms respond to some disadvantages to the use of cubic equations of state, and these inaccuracies are canceled by one other when the EoS's are employed for the calculation of fluid properties, specifically for the VLE densities. Researchers has emphasized considerably on modeling long and convex molecules in addition to modeling small and simple molecules. Based on theory of Prigogine ${ }^{7}$ and Flory ${ }^{8}$, an equation for molecules treated as chains of segments, which is called Perturbed-Hard-Chain-theory (PHCT) was expressed by Beret and Prausnitz ${ }^{9}$, Donohue and Prausnitz ${ }^{10}$ and Gross and Sadowski ${ }^{11}$. Large nonspherical molecules such as heavy hydrocarbons and polymers are theoretically modeled as chain like molecules. A chain of tangent hard spheres is the simplest model of this type of molecule. Relaxation of tangency constraint in the tangent hard sphere chains by allowing adjacent hard spheres along the chain to overlap leads to a more realistic model which is known as fused hard sphere chain. Conversely, in the limit, when the center to center reduced length tends to one, the EoS for a fused hard sphere chain reduce to an equation of state of thermodynamic perturbation theory for a tangent hard sphere chain ${ }^{12}$.

We have considered different forms for the repulsive part for equations of state ${ }^{13}$ of hard spheres such as vdW EoS or CSvdW EoS ${ }^{18}$ and also for the equation of state of hard convex body chains such as Boublik ${ }^{14-16} \mathrm{vdW}$ EoS. For the attractive part of these EoS's we considered the exponent term to the temperature to zero to reproduce the usual form of vdW or CSvdW or Boublik vdW EoS's. In this paper, the chemical potential for Boublik vdW EoS has been derived and used to predict the liquidvapour equilibrium densities of $n$-alkane fluids. The predictions corresponding to this group of equations of state are within a degree of quality similar to the widely used Carnahan-Starling equation.

This paper is organized as follows. In section 2, we present the expressions for HS and $\mathrm{HCB}$ chain EoS and the corresponding expressions for chemical potentials. This section also includes a table for the critical parameters for $n$-alkanes. In Section 3 the results, i.e. the diagram for reduced density vs reduced temperature have been plotted and compared with the available experimental data of $n$-alkane fluids. Conclusions are presented in section 4.

\section{Equations of state for HS and HCB fluids}

This section includes three groups of equations of state. The three groups are made on the basis of extensively used add-on separation method of compressibility factor ${ }^{17-20}$ as $Z=Z_{\text {rep }}+Z_{\text {att }}$. The functional dependency of temperature, as introduced by Redlich and Kwong ${ }^{5}$, has a power-law form $\mathrm{T}^{-\delta}$ for the attractive part of the EoS's. For the expression of repulsive part of the EoS's has any of the three forms vdW, CS and Boublik. Note that in all calculations we set up a new parameter $\delta$ as the temperature-exponent in the attractive term. Notice that the selection to set up $\delta=0$ regains the original EoS's. The critical parameters and the analytic expressions for the chemical potential have been determined for the three groups, which allowed us to obtain the VLE densities. Here, for simplicity in calculation we employed the dimensionless variable $y=b / 4 v$ in place of the molar volume, $v$ with $b$ being the co-volume. Here, $y=b / 4 v$ is known as packing fraction ${ }^{20-23}$.

As is well known, the chemical potential, $\mu$, can be analytically obtained through ${ }^{13}$ :

$\mu=\int \frac{b}{4 y}\left(\frac{\partial P}{\partial y}\right)_{T} d y+\varphi(T)$

Where, the function $\phi$ has the temperature dependency.

The compressibility factor is expressed as, $Z=\frac{b P}{4 y R T}$ where $\mathrm{R}$ is the molar universal gas constant. To 
obtain an analytical expression for $\mu$ from equation (1), one requires an analytic expression for the equation of state.

\section{The van der Waals (vdW- $\delta$ ) groups}

The compressibility factor for the $\mathrm{vdW}-\delta$ set of EoS's is written as

$$
Z=\frac{1}{1-4 y}-\frac{4 a y}{b R T^{b x}}
$$

For $\delta=0$, equn.(2)reproduces the vdW EoS.

The critical point conditions can be solved to yield the critical parameters:

$y_{c}=0.083333, Z_{c}=0.375, a=\lambda b R T_{c}^{i+z}$,

Where, $\lambda=3.375$.

Using eqns. (1), the expression obtained for chemical potential is:

$\mu(y, T)=\frac{R T}{1-4 y}-\frac{8 a y}{b T^{2}}+R T \log \left|-\frac{y}{1-4 y}\right|+\varphi(T)$,

The Carnahan-Starling-van der Waals (CSvdW- $\delta$ ) group

The $\mathrm{CSvdW}-\delta$ group assumes its form by replacing the classical vdW repulsive term in eqn. (2) with the CS hard sphere EoS. The functional dependency of temperature for the attractive term of the EoS remains unchanged as in eqn.(2).

The compressibility factor for CS vdW- $\delta$ EoS's can now be written as

$$
Z=\frac{1+y+y^{2}-y^{2}}{(1-y)^{2}}-\frac{4 a y}{b R T^{+2}}
$$

For $\delta=0$, equn.(4) reproduces the usual form of the CSvdW EoS [17].

Using eqns. (1), the expression obtained for chemical potential is:

$$
\mu(y, T)=\frac{R T(3-y)}{(1-y)^{2}}-\frac{8 a y}{b T^{2}}+R T \log (y)+\varphi(T) \text {. }
$$

The Boublik- van der Waals (Boublik vdW- $\delta$ ) group

Boublik proposed an equation of state for Hard Convex Bodies ${ }^{15}$ as

$Z_{k a b}=\frac{1+(3 \alpha-2) y+\left(3 \alpha^{2}-3 \alpha+1\right) y^{2}-\alpha^{2} y^{3}}{(1-y)^{3}}-\frac{4 a y}{b R T^{l+\delta}}$

Where, $\alpha$ is the nonsphericity parameter. When, $\alpha=1$, eqn.(6) reduces to Carnahan Starling equation. For, other hard convex body, $\alpha>1$. According to Boublik et al [15], the hard convex body equation can be extended to hard chain molecules of overlapping hard spheres $(0.5<L<1)$ or tangent hard spheres $(L=1)$ with formulation of parameter of nonspherecity as:

$$
\alpha=\frac{[1+(m-1) L][2+(m-1) L]}{2+(m-1)\left(3 L-L^{3}\right)}
$$

Using equation (1), the expression obtained for chemical potential is:

$$
\begin{aligned}
& \mu(y, T)=\frac{R T\left(y^{2}-2 y+1\right)}{(1-y)^{3}}-\frac{\alpha^{2} R T\left(y^{2}-4 y+1\right)}{(1-y)^{3}}- \\
& \frac{3 \alpha R T(y-1)}{(1-y)^{3}}-\frac{8 a y}{b T^{3}}+R T\left(1-\alpha^{2}\right) \log (1-y)+
\end{aligned}
$$

$$
R T \log (y)+\varphi(T),
$$

For $\alpha=1$, equation (8) reproduces the same equation for the chemical potential as that of the CS EoS.

The non-sphericity of the molecule is modeled in terms of the m-segments of hard convex bodies. It forms a hard molecule with a chain of freely jointed hard convex bodies. Each n-alkane can be modeled as a chain of $m$-segments of hard HCB. For determination of m-segment value for each n-alkane, one should comprehend that there should be some fundamental phial of our couch of $\mathrm{m}$ based on the nature of the HCB. For example, pentane is structured as $\mathrm{CH}_{3} \mathrm{CH}_{2} \mathrm{CH}_{2} \mathrm{CH}_{2} \mathrm{CH}_{3}$. One may accept that the base unit for pentane is $\mathrm{CH}_{2} \mathrm{CH}_{2}$, which could be approximately modeled as ethane $\left(\mathrm{CH}_{3} \mathrm{CH}_{3}\right)$. Since, two atoms of carbon cannot be approximated as one atom of carbon because of its 
higher mass as compared to the mass of hydrogen. But for lighter hydrogen one can approximate two hydrogen atoms as one atom or vice versa. Knowing the HCB of ethane and using this base of HCB with $\mathrm{m}=2.5$ to model Pentane. For decane, using the $\mathrm{CH}_{2} \mathrm{CH}_{2}$ as $\mathrm{HCB}, \mathrm{m}=5$. Note that the values guarded by the base model for the HCB have only sense in modeling the $n$-alkanes. Using this concept, the value of $m$ has been calculated for each $n$ alkane and is tabulated in Table 1. The table also gives the values

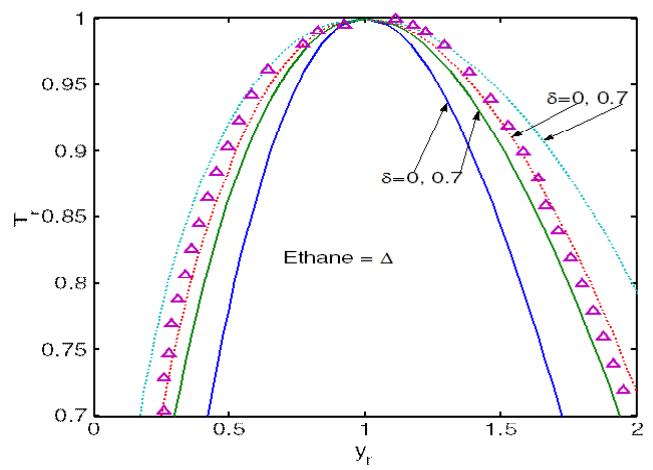

Fig.1: Plot of the reduced temperature versus reduced density for the VLE. The symbol represents data for Ethane [18]. The values are calculated from vdW- $\delta$ (continuous lines) and CS vdW- $\delta$ (dotted lines) or Boublik vdW- $\delta$ [for $m=1$ and $\alpha=1$ ].



Fig. 3: Plot of the reduced temperature versus reduced density for the VLE. The symbol represents data for Decane [18]. The values are calculated from $\mathrm{vdW}-\delta$ (continuous lines) and Boublik vdW- $\delta$ (dotted lines) [for $\mathbf{m}=\mathbf{5}$ and $\alpha=3$ ]. of the non-sphericity parameter $\alpha$ as calculated using equation (7).

For n-alkanes, when $\delta=0$ the critical point conditions can be solved to yield the critical parameters. The calculated critical parameters for pressure, temperature and volume for selected n-alkanes are:

$P_{c r}=\frac{1.20137 a}{17 b^{2}}, \quad T_{c r}=\frac{6.41435 a}{17 b R}, \quad V_{c r}=1.91653 b$

(Ethane)

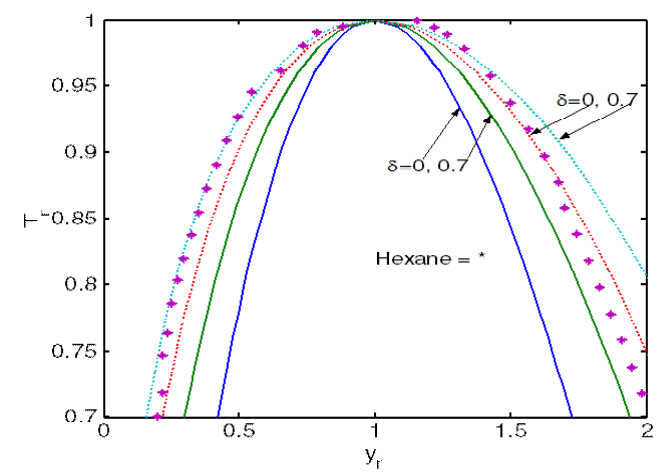

Fig. 2: Plot of the reduced temperature versus reduced density for the VLE. The symbol represents data for Hexane [18]. The values are calculated from $\mathrm{vdW}-\delta$ (continuous lines) and Boublik vdW- $\delta$ (dotted lines) [for $m=3$ and $\delta=2$ ].

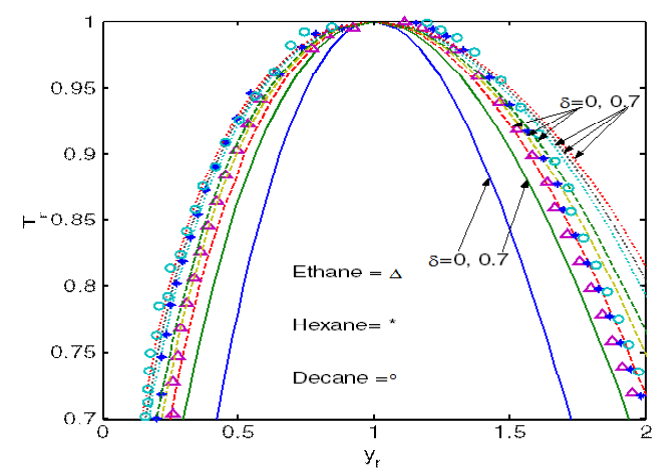

Fig. 4: Plot of the reduced temperature versus reduced density for the VLE. The symbols represent data for Ethane, Hexane and Decane [18]. The values are calculated from $\mathrm{vdW}-\delta$ (continuous lines) and Boublik vdW- $\delta$ (dashed lines for $\delta=0$, and dotted lines for $\delta=0.7$ ) [when, $\mathbf{m}=\mathbf{1 , 3}, \mathbf{5}$ and $\alpha=1,2,3$ ]. 
Table 1: Values of the critical and the nonsphericity parameter for a given НСB segment

\begin{tabular}{lccccc}
\hline n-alkanes & $\mathbf{m}$ & $\alpha$ & $\boldsymbol{y}_{\mathrm{c}}$ & $\boldsymbol{z}_{\boldsymbol{c}}$ & $\lambda$ \\
\hline Ethane & 1 & 1 & 0.13044408 & 0.358955 & 2.650308 \\
Pentane & 2.5 & 1.75 & --- & --- & --- \\
Hexane & 3.0 & 2 & 0.08962854 & 0.352899 & 4.235187 \\
Heptane & 3.5 & 2.25 & --- & --- & --- \\
Octane & 4.0 & 2.50 & --- & --- & --- \\
Nonane & 4.5 & 2.75 & --- & 0.349386 & 5.780184 \\
Decane & 5.0 & 3 & 0.0692945 & & \\
\hline
\end{tabular}

$P_{c r}=\frac{364.365 a}{12197 b^{2}}, \quad T_{c r}=\frac{2879.92 a}{12197 b R}, \quad V_{c r}=2.78929 b$ (Hexane)

$P_{c r}=\frac{483.575 a}{28863 b^{2}}, \quad T_{c r}=\frac{4993.44 a}{28863 b R}, \quad V_{c r}=3.60779 b$ (Decane)

\section{RESULTS AND DISCUSSION}

We examined the behavior of the three groups of EoS's by calculating the VLE densities, and compared them with the experimental data ${ }^{19}$ for fluids of n-alkanes. In particular, we calculated the co-existence curves in the diagram. The calculations were made by taking that we had observed to include the best choice for comparison with real data. Figs. (1)-(3) represents the results for the VLE densities (in reduced units) versus the reduced temperatures. The points represent the experimental data for $n$-alkanes (ethane, hexane and pentane) fluids ${ }^{19}$, and the lines represent the predictions obtained with the $v d W-\delta$, Boublik vdW- $\delta$ groups of EoS's. Note that figures (1)-(3) have been drawn separately for clear representation of the data lines and the available experimental points of selected n-alkanes. Figure 4 simply represents all the three figures (1)-(3) together in order to get a close difference between them. In figures (1)-(3), the continuous lines represent the groups belonging to the classical $v d W-\delta$ repulsive term and the dotted lines represent the groups belonging to the Boublik repulsive term. In figure 1, note that the dotted lines reproduce the data for CS EoS for $\mathrm{m}=1$ and $\alpha=1$. Figure 4 represents co-existence curves in the diagram with the EoS's for the values of exponent and the experimental points of the VLE densities for selected n-alkane fluids. In figure 4, for representing lines for exponent continuous lines are used (classical vdW- $\delta$ ). For Boublik vdW- $\delta$, the lines for exponent are represented by dashed lines and the lines for exponent are represented by dotted lines. From these observations it is clear that the modifications to temperature dependence i.e., by varying the exponent of the attraction term improve the result of prediction of saturation densities.

\section{Diagrams}

However, the co-existence curves of figure 4 shows that the saturation densities calculated with the classical vdW EoS $(\delta=0)$ are unsatisfactory. From the analysis of these coexistence curves it is evident that the use of CS EoS for the repulsive term only improves the results on the liquid branch over a small range of temperature. However, the plot of the theoretical liquid saturation densities is certainly different from that of the experimental points corresponding to both branches of the coexistence curve. It is clear that, by taking the several exponents in the CS vdW EoS or Boublik vdW- $\delta$, one can observe both the liquid and vapour branches in good correspondence with the experimental points for fluids, but this is deviational for the case of the classical vdW- $\delta$ groups. We conclude from this result that for each vdW- $\delta$ of EoS's group there must be a best value for the exponent for each fluid. Our study for simple fluids of $n$-alkanes, we may consider the best value to be in the range (0-1). 


\section{CONCLUSIONS}

In this paper, equations for chemical potential have been derived. These equations have been utilized in finding the VLE density of HCB chain and HS systems. All the three groups of EoS's [24-30] have been studied by finding the liquidvapour equilibrium densities of $n$-alkane fluids. The observations clearly indicates that the EoS's obtained by add-on separation method of compressibility factor $^{20-21}$ as , have predicted the experimental data closely. The predictions of VLE densities for these fluids can be numerically improved by finding the best value of the exponent for each case. The better results in the work related to mixtures and mixing rules can be obtained if a most suitable choice for the - exponent value becomes available.

\section{ACKNOWLEDGEMENTS}

One of us (B.P.A) specially thanks the University Grant Commission, Government of India for financial support (PSJ-001/13-14).

\section{REFERENCES}

1. Yelash, L.V.; and Kraska,T. Fluid Phase Equilib. 1999, 162, 115-130.

2. Valderrama, J.O. Ind. Eng. Chem. Res. 2003, 42,1603-1618.

3. Wei, Y.S.; Sadus, R.J. AlChE J. 2000, 46(1),169-196.

4. Esmaeilzadeh, F.; Roshanfeker, M. Fluid Phase Equilib. 2006, 239, 83-90.

5. Redlich, O.; Kwong, J.N.S. Chem. Rev. 1949, 44, 233-244.

6. Bertacco, A.; Elvassore, N.; Fermeglia, M.; Prausnitz, J.M. Fluid Phase Equilib. 1999, 158,183-191.

7. Prigogine, I. The Molecular Theory of Solutions, North-Holland, Amsterdam,1957.

8. Flory, P.J. J.Am. Chem. Soc. 1965, 87, 18331838.

9. Abrams, D.S.; Praunitz, J.M. A/ChE J. 1975, 21,116-128.

10. Donohue, M.D.; Prausnitz, J.M. AlChE J. 1978, 24(5), 849-860.

11. Gross, J.; Sadowski, G. Ind. Eng. Chem.Res. 2001,40(4),1244-1260.

12. Waziri, S. M.; Hamad, E. Z. Ind.Eng.Chem. Res. 2008, 47, 9658-9662.

13. Roma'n, F.L.; Mulero, A.; F. Cuadros, F. Phys . Chem. Chem. Phys. 2004, 6, $5402-5409$.

14. Boublik, T. J. Chem. Phys. 1975, 63, 40844085.

15. Boublik, T.; Vega, C.; Pena, M.D. J. Chem. Phys. 1990, 93,730-736.

16. Carnahan, N.F.; Starling,K.E. AIChE J. 1972 , 18, 1184-1189.
17. Carnahan, N.F.; Starling, K.E. J.Chem. Phys 1969, 51, 635-636.

18. Lemmon, E.W.; McLinden, M.O.; Fiend, D.G. Thermophysical Properties of Fluid Systems in NIST Chemistry WebBook, NIST Standard Reference Database n. 69, ed. Linstrom, P.J.; Mallard, W.G. National Institute of Standards and Technology (NIST), Gaithersburg, M.D.,http://webbook.nist.gov.

19. Sadus, R.J. J. Chem. Phys. 2001, 115, 14601462.

20. Sadus, R.J. J. Chem. Phys. 2002, 116, 59135913.

21. Sadus, R.J. Phys. Chem. Chem. Phys. 2002, 4, 919-921.

22. Peng, D.Y.; Robinson, D.B. Ind. Eng. Chem. Fundam. 1976, 15, 59-64.

23. Mathias, P.M.; Naheiri, T.; Oh, E.M. Fluid Phase Equilib. 1989, 47, 77-87.

24. Song, Y.; Hino,T.; Lambert, S.M.; Prausnitz,J.M. Fluid Phase Equilib. 1996, 117, 69-76.

25. Mulia, K.; Yesavage,V.F. Fluid Phase Equilib. 1989, 52, 67-74.

26. Walsh, J.M.; Gubbins, K.E. J. Phys. Chem. 1990, 94, 5115-5120.

27. Mohsen-Nia, M.; Modarressa, H.; Mansoori, G.A. Fluid Phase Equilib. 2003, 206, 2739.

28. Mathias, P.M. Ind. Eng. Chem. Res. 2003, 42, 7037-7044.

29. Wang, H.T.; Tasi, J.C.; Chen, Y.P. Fluid Phase Equilib. 1997, 138, 43-59.

30. Bertacco, A.; Elvassore, N.; Fermeglia, M.; Prausnitz, J.M. Fluid Phase Equilib. 1999, 158, 183-191. 